\title{
Memory-guided saccade abnormalities in schizophrenic patients and their healthy, full biological siblings
}

\author{
S. Landgraf ${ }^{1,2 *}$, I. Amado ${ }^{1}$, M.-C. Bourdel ${ }^{1}$, S. Leonardi ${ }^{1}$ and M.-O. Krebs ${ }^{1}$ \\ ${ }^{1}$ INSERM U796, Physiopathology of Psychiatric Diseases, University Paris René Descartes, Faculty of Medicine, Sainte Anne Hospital, Paris, \\ France \\ ${ }^{2}$ Humboldt University Berlin, Institute of Psychology, Berlin, Germany
}

Background. Ocular-motor inhibition errors and saccadic hypometria occur at elevated rates in biological relatives of schizophrenic patients. The memory-guided saccade (MS) paradigm requires a subject to inhibit reflexive saccades (RSs) and to programme a delayed saccade towards a remembered target.

Method. MS, RS, and central fixation (CF) tasks were administered to 16 patients who met the criteria for DSM-IV schizophrenia, 19 of their psychiatrically healthy siblings, and 18 controls.

Results. Patients and siblings showed elevated MS error rates reflecting a failure to inhibit RSs to a visible target, as required by the task. In contrast to controls, prior errors did not improve MS accuracy in patients and siblings.

Conclusions. The specific characteristics of the elevated MS error rate help to clarify the nature of the disinhibition impairment found in schizophrenics and their healthy siblings. Failure to inhibit premature saccades and to improve the accuracy of subsequent volitional saccades implicates a deficit in spatial working-memory integration, mental representation and/or motor learning processes in schizophrenia.

Received 26 March 2007; Revised 15 August 2007; Accepted 24 August 2007; First published online 2 November 2007

Key words: Error rate, memory-guided, saccades, schizophrenia, siblings, working memory.

\section{Introduction}

Schizophrenic patients have repeatedly shown impaired inhibition of reflexive (externally triggered) saccades and execution of volitional (internally triggered) saccades (Calkins \& Iacono, 2000; McDowell \& Clementz, 2001). Whether the same deficits are also present in their biological relatives, possibly representing 'endophenotypes' (Gottesman \& Gould, 2003), is unclear (Curtis et al. 2001a,b; Karoumi et al. 2001; Ettinger et al. 2004, 2006). Levy et al. (2004) challenged the hypothesis (of Calkins et al. 2004) that volitional saccadic performance is abnormal in schizophrenic patients and their relatives by arguing that asymmetrical exclusion criteria for relatives and controls are predominantly responsible for heterogeneity between groups. In the reflexive saccade (RS) task, where inhibition demands are almost absent, and in the central fixation (CF) task, where inhibition demands are maximized, both schizophrenic patients (Fukushima et al. 1988, 1990a,b, 1994; Clementz et al.

* Address for correspondence: S. Landgraf, M.A., Service Hospitalo Universitaire, Hôpital Sainte-Anne, 7 rue Cabanis, 75014 Paris, France.

(Email: landgras@cms.hu-berlin.de)
1994; Sereno \& Holzman, 1995; Hutton et al. 2001; Krebs et al. 2001; Raemaekers et al. 2002; Boudet et al. 2005) and their siblings (Karoumi et al. 2001; Ettinger et al. 2004; Raemaekers et al. 2006) show normal performance. The memory-guided saccade (MS) paradigm consists of moving the eyes to a remembered location of a briefly flashing stimulus after a delay period. Specific characteristics, such as latency or accuracy, of the elicited saccade might depend on whether or not the subject commits a premature erroneous saccade towards the stimulus, a so-called disinhibition error. Indeed, the MS paradigm allows inhibitory processes (e.g. inhibiting an RS to the target) and volitional saccade initiation (e.g. generating a saccade to the remembered target location) to be decoupled. Therefore, two promising 'endophenotype' candidates in schizophrenia, namely elevated disinhibition error rate and hypometria (spatially inaccurate volitional saccades), can be investigated separately within one paradigm: the MS paradigm.

Elevated MS error rate has been reported in patients (Crawford et al. 1995; McDowell \& Clementz, 1996, 2001; Radant et al. 1997), independently of medication status (Müller et al. 1999), and in their relatives (Park 
et al. 1995; McDowell et al. 1996). Abel \& Douglas (2006) and McDowell \& Clementz $(1996,2001)$ have suggested a latency subclassification of those inappropriate MSs during the delay period, with the cut-off point between 'reflexive' MS errors (immediately after target presentation) and 'saccadic anticipation' MS errors (immediately before the signal to execute the volitional saccade) varying from 260 to $400 \mathrm{~ms}$ after stimulus onset. Other studies (Amador et al. 1995; Curtis et al. 2001b) have reported that high inhibitory conditions, for example the presence of distracters, proved to be highly specific in identifying a vulnerability among families with cases of schizophrenia. Patients with a family history of schizophrenia also showed deficits at smaller angles than patients without a family history of this disease (Schwartz et al. 1995). The percentage of 'reflexive' MS errors might, therefore, yield higher specificity in detecting a marker of vulnerability in schizophrenia with increasing target amplitude.

Supporting this view is the observation of dysmetric MSs in schizophrenic patients (Fukushima et al. 1990a ; Crawford et al. 1995; McDowell \& Clementz, 1996, 2001; Karoumi et al. 1998) and their relatives (Park et al. 1995; Ross et al. 1998; McDowell et al. 2001). McDowell et al. (2001) found an increase in accuracy in schizophrenic patients, their relatives and controls when comparing MS trials without error to all MS trials. According to the authors, the premature erroneous MSs "provide "practice" that improves the accuracy of the eventual memory saccades'. However, it could be argued that patients and their relatives would not benefit equally from such a 'practice' effect if their errors carried little spatial information or if additional target or motor command information was not integrated sufficiently into working memory.

To contribute to the discussion on possible ocularmotor susceptibility markers for schizophrenia, we addressed the following questions. First, we chose to include only full biological adult siblings of schizophrenic patients in order to decrease heterogeneity in the relative group. Second, we expected that the MS paradigm measures would best discriminate between groups, as performance measures in the RS and CF typically do not. We hypothesized that (a) patients and their siblings would show an elevated MS error rate consisting of more 'reflexive' than 'saccadic anticipation' errors, $(b)$ this inhibition impairment would become more pronounced with increasing target amplitude, and (c) the increase in accuracy from MS trials without error to MS trials with error would be less pronounced in patients and their siblings. Third, to ensure that different exclusion criteria for siblings and the control group do not account for
Table 1. Demographic information for the participant groups

\begin{tabular}{lclll}
\hline Group & Gender & $\begin{array}{l}\text { Age } \\
\text { (years) }\end{array}$ & $\begin{array}{l}\text { Educational } \\
\text { level }^{\mathrm{a}}\end{array}$ & $\begin{array}{l}\text { Handedness } \\
(\%)\end{array}$ \\
\hline $\mathrm{C}$ & $3 \mathrm{~F}$ & $27.3(4.04)$ & $1.67(0.58)$ & $66 \mathrm{R}, 33 \mathrm{X}$ \\
& $15 \mathrm{M}$ & $25.5(3.29)$ & $2.13(1.19)$ & $66 \mathrm{R}, 17 \mathrm{~L}, 17 \mathrm{X}$ \\
$\mathrm{FS}$ & $13 \mathrm{~F}$ & $26.5(6.51)$ & $1.73(0.79)$ & $77 \mathrm{R}, 8 \mathrm{~L}, 15 \mathrm{X}$ \\
& $6 \mathrm{M}$ & $26.5(2.81)$ & $2.00(1.00)$ & $66 \mathrm{R}, 34 \mathrm{X}$ \\
$\mathrm{SZ}$ & $3 \mathrm{~F}$ & $28.7(4.16)$ & $1.67(0.58)$ & $66 \mathrm{R}, 34 \mathrm{X}$ \\
& $13 \mathrm{M}$ & $25.6(4.05)$ & $2.08(1.08)$ & $62 \mathrm{R}, 38 \mathrm{X}$ \\
\hline
\end{tabular}

C, Controls; FS, full siblings; SZ, schizophrenic patients; F, female; $\mathrm{M}$, male; R, right-handed ; L, left-handed ; $\mathrm{X}$, mixed.

Values are given as mean (standard deviation).

a $1=$ more than 3 years of post-high-school education; $2=$ less than 3 years of post-high-school education; $3=$ High School Diploma (Baccalaureat) $; 4=$ high school not finished.

performance differences, we selected families where the schizophrenic patient was the only psychopathological case up to second degree of their family. We also tested for intra-family correlations for all measurements.

\section{Method}

\section{Participants}

Sixteen clinically stabilized schizophrenic in- and outpatients (SZ, 13 males) fulfilling DSM-IV criteria (APA, 1994) and 19 of their full biological siblings (FS, six males; 13 patients had one participating sibling, three patients had two) participated. Families were recruited in the Department of Psychiatry at the University Unit of Sainte-Anne Hospital (SHU), Paris, France. The patient was the only family member (up to second degree) affected by a psychiatric disorder. Eleven patients were receiving antipsychotic medication. Five were on classical antipsychotics (chlorpromazine equivalent: $506 \mathrm{mg} /$ day, S.D. $=358$ ) and six were on atypical antipsychotics (chlorpromazine equivalent: $600 \mathrm{mg} /$ day, S.D. $=184$ ) (Rijcken et al. 2003). In the case of concomitant mood stabilizer treatments, families were excluded from the study. Eighteen healthy volunteers (C, 15 males) were recruited from advertisement and personal references and were matched in age, gender and educational level to SZ. Exclusion criteria for all groups encompassed ophthalmological/neurological/cardiovascular diseases, substance abuse or dependence (past or present), severe extrapyramidal symptoms, a former head trauma, or birth complications. Demographic information for all three groups is summarized in Table 1 for males and females separately. 
The local ethical committee (Comité Consultatif de Protection des Personnes se prêtant à une Recherche Biomedicale) approved the study and all subjects gave written informed consent prior to participation. FS and $\mathrm{C}$ were paid 30 Euros for participating in the study.

\section{Procedure}

SZ completed the diagnostic assessment and the ocular-motor test battery within 2.5 days, FS and C within 1. Tests were conducted from 09:00 to 13:00 hours.

\section{Diagnostic assessment}

Diagnoses were based on DSM-IV criteria (APA, 1994). Trained psychiatrists (M.-O.K., I.A., S.L.) administered the Diagnostic Interview for Genetic Studies-3rd Version (DIGS-III; Nürnberger et al. 1994, French translation: Krebs et al. personal communication) to SZ and FS to attest schizophrenia diagnoses and to rule out a personal and family history of psychiatric diseases, and the Structured Clinical Interview for DSM III-R - Non-Patient Edition (SCID-NP; Spitzer et al. 1990) to screen C. The same exclusion criteria were applied to FS and C so that the former differed only in having a first-degree relative diagnosed with schizophrenia. Interviews covered each participant's whole lifetime. Interviewers were not blind to groups but the persons analysing the ocular-motor tracks were blind to the diagnosis status. Handedness was tested specifically during a neurological assessment adapted from the Edinburgh assessment (Krebs et al. 2000).

\section{Eye movement recording}

An infra-red oculographic device (IRIS, Scalar) recorded eye movements using iris/sclera reflection $\left(5 \mathrm{~V}, 1 \mathrm{~min}\right.$ resolution, linearity $0 \pm 25^{\circ}$; Reulen et al. 1988). While sitting on a chair in a darkened room, the head of the participant was positioned on a chin-rest $1.5 \mathrm{~m}$ in front of a light emitting diode bar. Data acquisition software (developed in our laboratory by J. Serran and D. Vital) with a sampling frequency of $500 \mathrm{~Hz}$ administered pre-programmed visual sequences.

\section{Ocular-motor paradigms}

The results of three of the ocular-motor tasks are presented here. Tasks consisted of three trials with 10 stimuli each. In the RS task, participants had to follow the targets with their eyes as fast and as accurately as possible. After a 2-s central fixation phase, pseudo-random stimuli (eccentricities: $\pm 4^{\circ}, 6^{\circ}, 8^{\circ}, 10^{\circ}$ or $12^{\circ}$; inter-stimulus interval $2-2.8 \mathrm{~s}$ ) were presented for $800 \mathrm{~ms}$. During the CF task, participants were instructed to look at the central light for $45 \mathrm{~s}$ while distracters (same as RS task stimuli) were presented. In the MS task, participants first had to fixate on the central light. After $900-1300 \mathrm{~ms}$ a target (same as RS task stimuli) appeared for $200 \mathrm{~ms}$. Central light extinction 1800-2200 ms after stimulus presentation signalled movement of the eyes to the memorized target location. Reappearance of the central light initiated the next trial (Fig. 1a).

\section{Measurements}

Independent scorers, unaware of subjects' group status, recorded a total of 3539 saccades defined as having a velocity between $60 \%$ s and $800^{\circ} / \mathrm{s}$ and a primary gain between 0.2 and 2.0. We measured saccade latency (time between target onset and eye movement initiation) and primary and final gain (eye movement amplitude/target eccentricity) in the RS and the MS paradigm as well as error rate (number of stimuli presentations with an inappropriate saccade towards the target/total number of stimuli) in the CF and MS paradigm. Errors were inappropriate saccades towards the distracter (CF) or the to-be-memorized stimulus (MS) and had a latency of more than $60 \mathrm{~ms}$ after stimulus onset (CF and MS task) until $60 \mathrm{~ms}$ after central light offset (MS task only) (Fischer, 1987).

\section{Statistical analyses}

SPSS version 12.0.1 (SPSS Inc., 2003) was used. Data distributions fulfilled, if not specified otherwise, the criteria for the statistical procedures. All analyses of variance (ANOVAs) included one random factor 'family'. To investigate possible confounding effects of gender and side, we conducted a preliminary threefactor ANOVA with two between-group factors, group (SZ, FS, C) and gender (male, female), and one within-group factor, side (saccade to the right or left). Then, group differences for the variables were tested using a univariate one-way ANOVA with separate analyses for the different trials in the MS task (with, without error). Post-hoc Tukey $t$ tests were performed for pairwise group comparisons. To compare variables between trials in the MS task (with, without error), we conducted repeated-measures $t$ tests for each group. To test for intra-family correlations, we used the intra-class correlation method, using 'within' and 'between' family variances. The significance level was $5 \%$. 


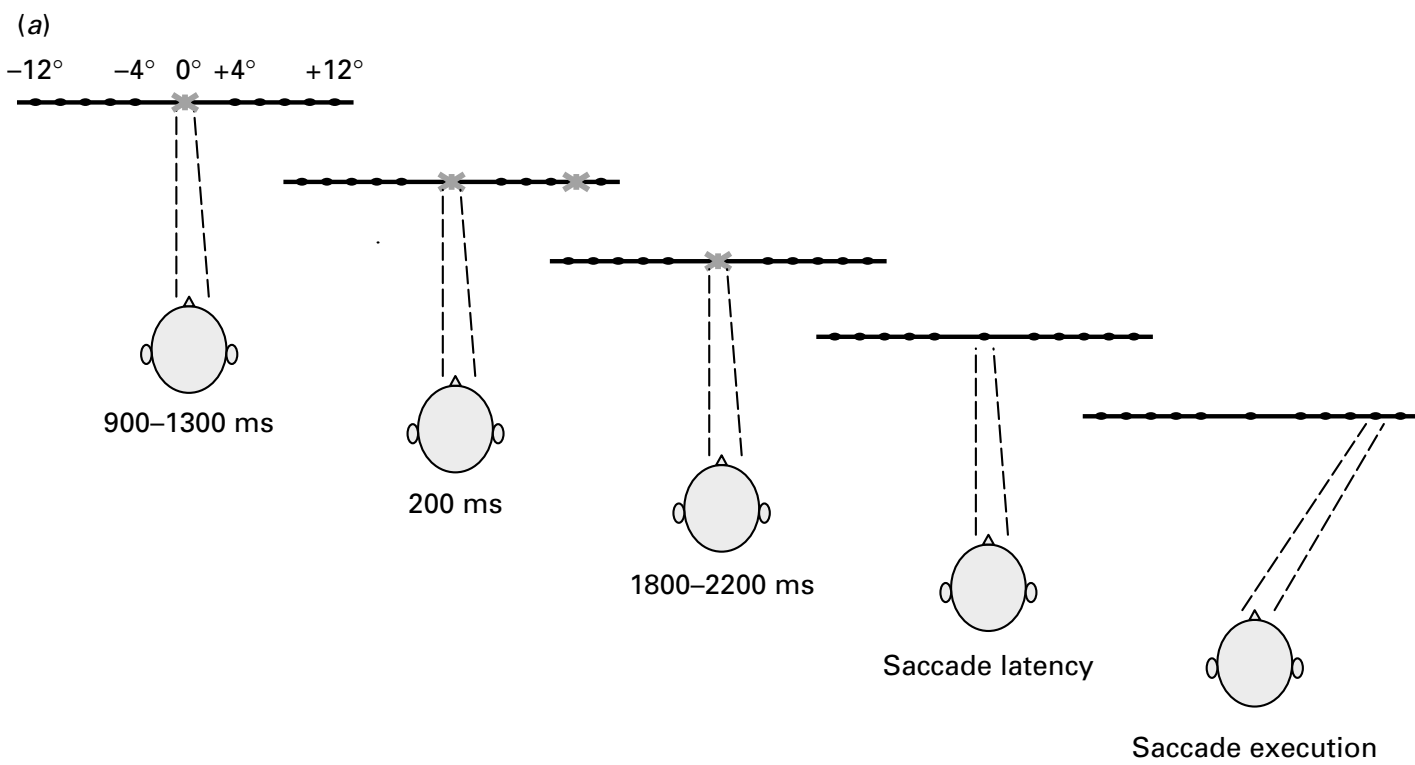

(b)

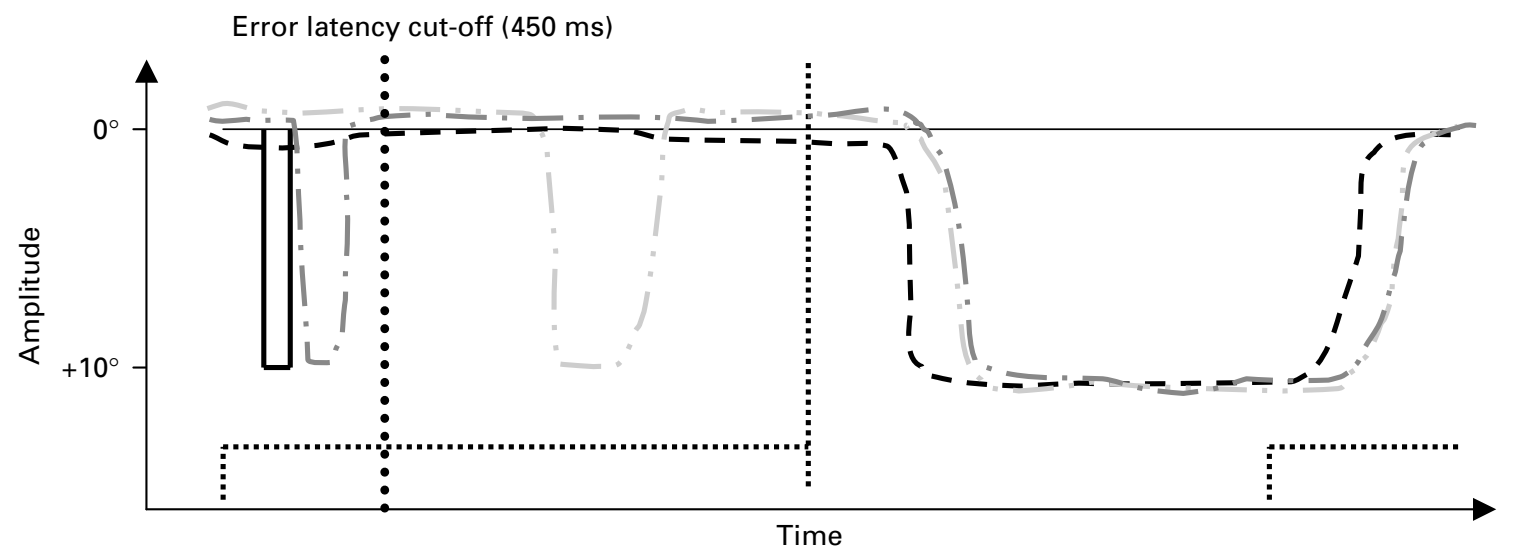

Fig. 1. The memory-guided saccade (MS) paradigm. (a) MS sequence with a target at $10^{\circ}$ to the right of the central fixation point. (b) Examples of normal and abnormal performance in the MS paradigm. - _ — , MS trial without error ; - : - , MS trial with 'reflexive' error $(\mathrm{RE}) ;-\ldots$, , MS trial with 'saccadic anticipation' error (SAE) ; $\_$, target flash; $\ldots . . . . .$. central light activation.

The demands to inhibit premature saccades to a target in the MS task differ with respect to the time elapsed after stimulus presentation (McDowell \& Clementz, 1996, 2001; Abel \& Douglas, 2006). As the MS error latency distribution was bimodal with a point of scarcity at $450 \mathrm{~ms}$, we subcategorized (cut-off $=450 \mathrm{~ms}$ ) MS errors into 'reflexive errors' (REs) and 'saccadic anticipation errors' (SAEs) (Fig. 1b). We compared the relative amounts of REs and SAEs between groups using a univariate oneway ANOVA and post-hoc Tukey $t$ tests. To test whether absolute stimulus amplitude would have an impact on error type, we conducted $\chi^{2}$ tests for 'small stimulus amplitude' $\left(4^{\circ}, 6^{\circ}, 8^{\circ}\right)$ and 'large stimulus amplitude' $\left(10^{\circ}, 12^{\circ}\right)$ independently for REs and SAEs.

\section{Results}

\section{Preliminary analyses}

Groups did not differ in age $[F(2,50)=0.113, p=0.893]$, educational level $[F(2,46)=0.272, p=0.763]$ or handedness $[F(2,50)=0.841, p=0.437]$. FS had more females $(68 \%)$ than SZ (19\%) and C (16\%). The Kolmogorov-Smirnov test showed that variables for latency and gain were normally distributed in all groups. As the distributions of MS and CF error 
Table 2. Means, standard deviations (in parentheses), and effect sizes for latency and gain in the reflexive and memory-guided saccade paradigm

\begin{tabular}{llllllll}
\hline & Group & $n$ & $\begin{array}{l}\text { Latency } \\
(\mathrm{ms})\end{array}$ & $\begin{array}{l}\text { Primary gain } \\
(\%)\end{array}$ & $\begin{array}{l}\mathrm{ES}_{\text {Primary gain }} \\
(d)\end{array}$ & $\begin{array}{l}\text { Final gain } \\
(\%)\end{array}$ & $\begin{array}{l}\mathrm{ES}_{\text {Final gain }} \\
(d)\end{array}$ \\
\hline Reflexive saccades & $\mathrm{C}$ & 18 & $179.4(20)$ & $96.9(12)$ & - & $98.6(10)$ & - \\
& FS & 19 & $169.3(29)$ & $98.4(9.2)$ & - & $98.3(8.7)$ & - \\
& SZ & 16 & $170.9(24)$ & $92.1(17)$ & - & $96.3(17)$ & - \\
Memory-guided saccades & C & 18 & $307.8(58)$ & $89.5(12)^{\mathrm{b}}$ & 0.73 & $92.5(16)^{\mathrm{c}}$ & 0.72 \\
(without error) & FS & 19 & $290.7(50)$ & $82.4(21)$ & - & $88.9(21)$ & - \\
& SZ & 15 & $335.9(104)$ & $81.3(17)$ & - & $86.9(12)$ & - \\
Memory-guided saccades & C & 16 & $379.4(133)$ & $100.8(26)^{\mathrm{b}}$ & - & $101.6(19)^{\mathrm{c}}$ & - \\
(with error) & FS & 19 & $365.7(110)$ & $81.8(22)^{\mathrm{a}}$ & 0.79 & $85.2(20)^{\mathrm{a}}$ & 0.84 \\
& SZ & 14 & $362.9(128)$ & $82.7(22)^{\mathrm{a}}$ & 0.75 & $86.8(22)^{\mathrm{a}}$ & 0.72 \\
\hline
\end{tabular}

C, Controls; FS, full siblings; SZ, schizophrenic patients; $n$, number of subjects; ES, effect size; $d$, Cohen's $d$.

Values within the reflexive saccade (RS) paradigm and, separately for trials with and without error, in the memory-guided

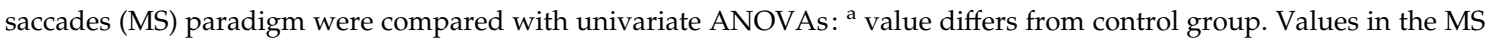
paradigm were compared between trials without error and trials with error separately for each group with repeated-measures $t$ tests: $b, c$ values differ between MS without and with errors.

rates in the MS paradigm were negatively skewed, we performed a square root transformation to ensure statistical validity. There was no difference in latency or accuracy either for gender or side. Therefore, both factors were collapsed for all further analyses. A summary of the results is presented in Table 2.

\section{Reflexive saccades}

Groups did not differ in latency $[F(2,50)=0.892$, $p=0.416]$ or in primary $[F(2,50)=1.133, p=0.330]$ or final gain $[F(2,50)=0.177, p=0.839]$.

\section{Central fixation}

Two SZ, $12 \mathrm{FS}$ and nine $\mathrm{C}$ had $0.0 \%$ errors. Groups did not differ in $C F$ error rate $[F(2,27)=2.296, p=0.120$; $C F$ error rates: SZ $11.6 \%$, FS 2.9\%, C 3.1\%].

\section{Memory-guided saccades}

\section{Error rate}

The data for one SZ had to be excluded because of technical difficulties. There was a main effect for group $[F(2,49)=11.648 \quad p<0.001]$. The subsequent $t$ tests showed that SZ $(31 \%, p<0.001$, Cohen's $d=1.7)$ and FS $(18.4 \%, p=0.012$, Cohen's $d=1.4)$ had an elevated error rate compared to C $(9.0 \%)$. SZ and FS did not differ $(p=0.129)$.

Comparing the relative amount of error type revealed a main effect for group $[F(2,49)=3.833$, $p=0.028$ ]. MS errors were mostly reflexive for SZ ( $76 \%$ RE, $p=0.034$, Cohen's $d=0.85)$ compared to C $(49 \%$

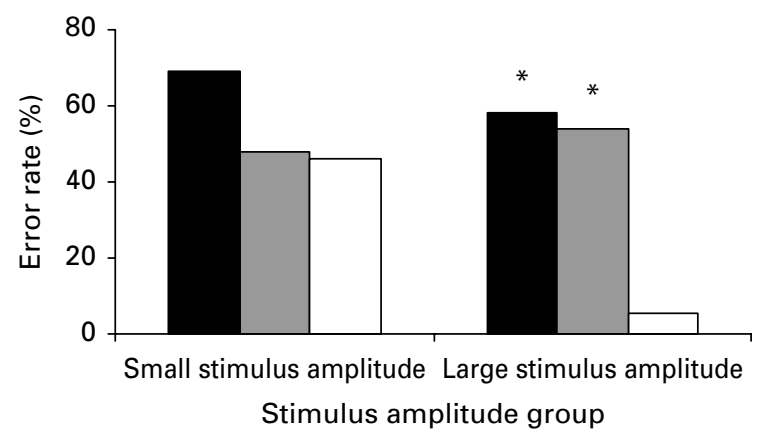

Fig. 2. Proportion of reflexive errors as a function of stimulus amplitude in the memory-guided saccade (MS) paradigm. Reflexive error rate remained high for patients ( and siblings $(\square)$ regardless of stimulus amplitude, whereas the 'reflexive' errors levelled off in the control group $(\square)$ with increasing stimulus amplitude. Small stimulus amplitude $=4^{\circ}, 6^{\circ}, 8^{\circ}$; Large stimulus amplitude $=10^{\circ}, 12^{\circ}$. * Value differs from control group.

RE). FS showed a trend in the same direction as SZ, but the comparison with $\mathrm{C}$ did not yield significance (60\% RE, $p=0.095)$.

Testing the influence of stimulus amplitude on error type revealed that $\mathrm{RE}$ rate for $\mathrm{C}$ decreased from $46.2 \%$ at 'small stimulus amplitude' to $5.6 \%$ at 'large stimulus amplitude' $\left[\chi^{2}(\mathrm{df}=2)=12.787, p=0.001\right]$. However, the error rate for SZ and FS remained stable regardless of stimulus amplitude (SZ 68\% and 58\%, FS $48 \%$ and $54 \%$, respectively, Fig. 2). SAE rates did not differ between groups for 'small target amplitude' [SZ 29\%, FS 30\%, C 37\%, $\left.\chi^{2}(\mathrm{df}=2)=2.527, p=0.347\right]$ 
and 'large target amplitude' [SZ 29\%, FS 52\%, C 50\%, $\left.\chi^{2}(\mathrm{df}=2)=3.868, p=0.173\right]$.

\section{MS trials without error}

Groups did not differ either in latency $[F(2,49)=1.655$, $p=0.202]$ or in primary $[F(2,49)=1.160, p=0.320]$ or final gain $[F(2,49)=0.013, p=0.624]$.

\section{MS trials with error}

One SZ and two C had $0.0 \%$ errors and were excluded from latency and gain comparisons. There was a main effect for group in primary $[F(2,46)=3.405, p=0.042]$ and in final gain $[F(2,46)=3.233, p=0.049]$. Both SZ (primary gain $=81.8 \%$, final gain $=85.2 \%$ ) and FS $(82.7 \%, 86.8 \%)$ differed from C $(100.8 \%, 101.6 \%)$, although the subsequent $t$ tests did not yield the conventional $5 \%$ significance level $\left(0.05<p^{\prime}\right.$ s $\left.<0.1\right)$.

\section{MS trials with error vs. trials without error}

The within-group repeated-measures $t$ tests showed that neither SZ nor FS differed in their primary or final gain values between MS trials with and without error. However, $\mathrm{C}$ had smaller primary $(t=2.813, \mathrm{df}=15$, $p=0.013)$ and final $(t=2.780, \mathrm{df}=15, p=0.014)$ gains in MS trials without error compared to MS trails with error. Latency tended to be longer in MS trials with error in all groups but this difference did not reach significance.

\section{Intra-family correlations}

We calculated intra-family correlations for latency and gain in the RS and MS paradigm. None of these variables showed a significant correlation (data not shown). We also tested for intra-family correlation regarding the error rates in the $\mathrm{CF}$ and the MS paradigm. Neither test was significant $\left(r_{\mathrm{CF}}=-0.062, p=0.802\right.$; $\left.r_{\mathrm{MS}}=0.077, p=0.379\right)$.

\section{Discussion}

To investigate the usefulness of ocular-motor abnormalities as liability markers for schizophrenia, we tested schizophrenic patients and their biological siblings in three visual paradigms. The MS paradigm was more specific in identifying saccadic abnormalities, possibly indicating a marker for schizophrenia, than the RS or the CF task, where performance did not differ between groups. Increased MS error rate in the index groups (SZ and FS) was characterized by 'reflexive' errors. Moreover, 'reflexive' error rates were the same only in SZ and FS, whether the stimulus amplitude was small or large. Accuracy did not improve from MS trials without error compared to MS trials with error in SZ and FS, whereas it did in C. Finally, intra-family correlations for all variables were insignificant.

\section{Disinhibition and errors}

Supporting hypotheses $(a)$ and $(b)$, the results in the MS task are in line with former investigations (Crawford et al. 1995; Park et al. 1995; McDowell \& Clementz, 1996, 2001; Radant et al. 1997) and confirm the importance of disinhibition abnormalities as possible endophenotypes for schizophrenia. Recent debate has concentrated on antisaccade error rate (Calkins et al. 2004; Levy et al. 2004), which correlates highly with MS error rate (0.78 in SZ and 0.74 in relatives; McDowell et al. 2001). An important aspect of the MS paradigm is that inhibition and initiation processes are temporarily decoupled. Involuntary MS 'reflexive' errors are more prevalent in SZ and FS even at the largest target amplitudes $\left(10^{\circ}\right.$ and $\left.12^{\circ}\right)$ compared to $\mathrm{C}$, indicating a more specific deficit in the index groups. The reason for this difference is unknown but it may reflect heightened disinhibition immediately after stimulus onset. While committing 'reflexive' errors might be intrinsic to healthy and vulnerable subjects, greater target amplitude reveals decreased inhibition ability in SZ and FS. Furthermore, the three groups did not differ in 'saccadic anticipation' errors. This last point indicates that the temporal preparation of planned responses is not impaired. Taking into account specific characteristics of erroneous MSs (latency) and MS stimuli (amplitude) might, therefore, be of importance for understanding error rate heterogeneity in this paradigm in the current literature.

\section{Accuracy}

Confirming previous results of impaired MS accuracy (Crawford et al. 1995; Park et al. 1995; Karoumi et al. 1998; Ross et al. 1998; McDowell \& Clementz, 2001), we found hypometric primary and final gain during MS trials with error in SZ and FS. However, this was primarily due to improved accuracy in $C$ in trials with error, which was not observable in the index groups. This finding confirms our hypothesis that SZ and FS have difficulty in adjusting their accuracy, taking into account their previous errors. This finding contradicts the results of McDowell et al. (2001), who found improved accuracy in schizophrenic patients, their relatives, and controls in MS trails without error compared to all MS trials. One possible explanation for finding an increase in accuracy in our $\mathrm{C}$ group alone is that we compared trials with and without error directly, allowing us to maximize discrimination power between these two types of task fulfillment. 
A second possible reason, preventing SZ and FS from improving their accuracy in MS trials with error, might stem from the implicit consequences of an increased MS error rate for working memory and learning processes. Information theory postulates that a less probable event carries more information than a more probable event (Dayen \& Abbott, 2001). The index groups showed an elevated MS error rate, thus making it a more probable event. Hence, the more frequent MS errors of SZ and FS would carry less visuospatial information than the less frequent MS errors of C. Consequently, less visuospatial information, which might be needed, for example, to maintain target characteristics in working memory or to facilitate motor learning processes, would be available for SZ and FS.

The third explanation stems from the fact that making an error allows information to be obtained about the target through foveal (rather than parafoveal) vision. It is possible that controls might extract more information from their foveal vision than SZ or FS on the basis of a previous error. Indeed, a deficit in foveal perception has been demonstrated in schizophrenia and proposed as a marker of vulnerability (Korn, 2000).

A fourth possible explanation might be a poor mental representation of the to-be-memorized target location or the executed motor command in working memory. In the case of proper task fulfillment, target characteristics can be used by SZ, FS and C to perform equally accurate MSs. However, in the event of an unintentional error, additional information about the target and its location might not be integrated correctly into working memory or might interfere with motor response or motor learning processes in the index groups. SZ and FS seem to be more occupied with having executed the premature saccade than with being able to process target or motor command characteristics. It is also noteworthy that there was a trend for saccadic accuracy to be lower in patients and their siblings compared to controls in MS trials without error. However, this result did not yield significance because of the low statistical power. Impaired accuracy in MS trails with error and, simultaneously, an elevated MS error rate observed in SZ and FS, however, corroborate the liability hypothesis of a disinhibition deficit and working-memory integration impairment in schizophrenia. The poor integration or poor mental representation of unintentional errors in working memory resembles some abnormalities already observed using antisaccade tasks in schizophrenia. Indeed, using a paradigm of antisaccades under standard and precue conditions, Reuter et al. (2006) found enhanced error rates in patients with a poor integration of the task set generated by the antisaccade mechanism and poor awareness of error detection. The authors related these abnormalities to reflecting a more generalized impairment of self-monitoring in schizophrenia (Frith, 1992).

Finally, contributing to the view considering disinhibition deficit as an ocular-motor marker, Ettinger et al. (2006) found abnormalities in antisaccades with saccade hypometria in monozygotic co-twins discordant for schizophrenia. In addition, it has been largely demonstrated that the dorsolateral prefrontal cortex (DLPFC) is more particularly involved in the suppression of unwanted reflexive saccades (Ploner et al. 2005), in antisaccades and in MSs (Pierrot-Deseilligny et al. 2003).

\section{Familial risk}

In recent research investigating saccadic metrics as possible 'endophenotypes' for schizophrenia, a difference between the relatives of normally performing SZ and the relatives of abnormally performing SZ has been reported frequently (Crawford et al. 1998; Curtis et al. 2001a; Brownstein et al. 2003; Calkins et al. 2004), indicating that schizophrenia and ocular-motor abnormalities might represent genetically independent phenomena. Furthermore, spatial workingmemory deficits associated with the MS task might have a developmental component and be detectable in early childhood (Diwadkar et al. 2001). In this study, we did not find intra-family correlations for any of the variables, which might be explained by the restricted statistical power arising from the small sample size or from the fact that the reported abnormalities were in fact present in most of SZ and FS. We also only included families where the patient, treated with a single antipsychotic drug, was the only psychopathological case in the family.

We did not find impairment of reflexive saccades in SZ or in FS. Most of our group of patients were receiving medication. Reilly et al. (2005) observed shorter latencies in drug-naive schizophrenic patients for reflexive saccades while earlier literature did not (Broerse et al. 2001). As emphasized by these authors, the influence of treatment, disease chronicity or methodological conditions could explain this divergence. The confounding effect of treatment could also explain the absence of impairment in the central fixation paradigm, because in a previous study we observed an increased error rate in patients compared to controls, with non-treated schizophrenic patients (Krebs et al. unpublished observations).

Despite not being able to control for medication effects, we can say that MS error rate does not seem to 
depend on antipsychotic medication (Müller et al. 1999) and, more importantly, none of the siblings has taken any medication. Finally, we should mention that the cut-off point for 'reflexive' and 'saccadic anticipation' errors was data based and requires a more elaborate theoretical foundation derived from the work with healthy subjects and other psychopathological groups before results can be compared between studies.

\section{Conclusions}

Schizophrenic patients and their healthy siblings show a similar ocular-motor impairment pattern in the MS paradigm. The increased MS error rate indicates heightened disinhibition in the index groups. However, specific characteristics of MS errors might help to increase specificity in detecting saccadic impairment. Furthermore, the absence of an increase in accuracy in MS trials with error compared to MS trails without error in the index groups might be attributable to their altered ability to incorporate visuospatial information into working memory and/or to use this information to modify a previously planned saccade. Widely studied in non-human primates (e.g. Chafee \& Goldman-Rakic, 1998), the MS paradigm provides an excellent animal model of response inhibition and working memory and might therefore serve as a translational endophenotype (Gould \& Gottesman, 2005). Further research is necessary to assess mental representation deficits, cerebellar contribution and impairment specificity. Finding similar ocular-motor impairment patterns in SZ and FS indicates that assessing distinct behavioural measures, perhaps associated with DLPFC dysfunction (Pierrot-Deseilligny, 1994), might be useful in identifying liability loci for schizophrenia.

\section{Acknowledgements}

We thank Hernan Picard and Marianna Enamoneta for their helpful comments on the manuscript and Larry A. Abel for clarifying important methodological questions. Steffen Landgraf was supported by the 'Leonardo da Vinci' Grant and Sabinien Leonardi by the 'Fondation pour la Recherche Médicale'. The study was partly supported by the Lilly France Company and the Sainte-Anne Hospital, Paris, France.

\section{Declaration of Interest}

None.

\section{References}

Abel LA, Douglas J (2006). Effects of age on latency and error generation in internally mediated saccades. Neurobiological Aging 28, 627-637.

Amador XF, Malaspina D, Sackeim HA, Coleman EA, Kaufmann CA, Hasan A, Gorman JM (1995). Visual fixation and smooth pursuit eye movement abnormalities in patients with schizophrenia and their relatives. Journal of Neuropsychiatry and Clinical Neurosciences 7, 197-206.

APA (1994). Diagnostic and Statistical Manual of Mental Disorders, 4th edn. American Psychiatric Press: Washington, DC.

Boudet C, Bocca ML, Chabot B, Delamillieure P, Brazo P, Denise P, Dollfus S (2005). Are eye movement abnormalities indicators of genetic vulnerability to schizophrenia? European Psychiatry 20, 339-345.

Broerse A, Crawford T, den Boer J (2001). Parsing cognition in schizophrenia using saccadic eye movements: a selective overview. Neuropsychologia 39, 742-756.

Brownstein J, Krastoshevsky O, McCollum C, Kundamal S, Matthysse S, Holzman PS, Mendell NR, Levy DL (2003). Antisaccade performance is abnormal in schizophrenia patients but not in their biological relatives. Schizophrenia Research 63, 13-25.

Calkins ME, Curtis CE, Iacono WG, Grove WM (2004). Antisaccade performance is impaired in medically and psychiatrically healthy biological relatives of schizophrenia patients. Schizophrenia Research 71, 167-178.

Calkins ME, Iacono WG (2000). Eye movement dysfunction in schizophrenia: a heritable characteristic for enhancing phenotype definition. American Journal of Medical Genetics 97, 72-76.

Chafee MV, Goldman-Rakic PS (1998). Matching patterns of activity in primate prefrontal area 8a and parietal area 7ip neurons during a spatial working memory task. Journal of Neurophysiology 79, 2919-2940.

Clementz BA, McDowell JE, Zisook S (1994). Saccadic system functioning among schizophrenia patients and their first-degree biological relatives. Journal of Abnormal Psychology 103, 277-287.

Crawford TJ, Haeger B, Kennard C, Reveley MA, Henderson L (1995). Saccadic abnormalities in psychotic patients. I. Neuroleptic-free psychotic patients. Psychological Medicine 25, 461-471.

Crawford TJ, Sharma T, Puri BK, Murray RM, Berridge DM, Lewis SW (1998). Saccadic eye movements in families multiply affected with schizophrenia: the Maudsley family study. American Journal of Psychiatry 155, 1703-1710.

Curtis CE, Calkins ME, Grove WM, Feil KJ, Iacono WG (2001a). Saccadic disinhibition in patients with acute and remitted schizophrenia and their first-degree biological relatives. American Journal of Psychiatry 158, 100-106.

Curtis CE, Calkins ME, Iacono WG (2001b). Saccadic disinhibition in schizophrenia patients and their firstdegree biological relatives. Experimental Brain Research 137, 228-236.

Dayen P, Abbott LF (2001). Theoretical Neuroscience: Computational and Mathematical Modeling of Neural Systems. MIT Press: Cambridge, MA. 
Diwadkar VA, Sweeney JA, Boarts D, Montrose DM, Keshavan MS (2001). Oculomotor delayed response abnormalities in young offspring and siblings at risk for schizophrenia. CNS Spectrums 6, 899-903.

Ettinger U, Kumari V, Crawford TJ, Corr PJ, Das M, Zachariah E, Hughes C, Sumich AL, Rabe-Heskethe S, Sharmaf T (2004). Smooth pursuit and antisaccade eye movements in siblings discordant for schizophrenia. Journal of Psychiatric Research 38, 177-184.

Ettinger U, Piccioni M, Hall MH, Schulze K, Toulopoulou T, Landau S, Crawford TJ, Murray RM (2006). Antisaccade performance in monozygotic twins discordant for schizophrenia: the Maudsley twin study. American Journal of Psychiatry 163, 543-545.

Fischer B (1987). The preparation of visually guided saccades. Reviews of Physiology, Biochemistry and Pharmacology 106, 1-35.

Frith CD (1992). The Cognitive Neuropsychology of Schizophrenia. Erlbaum: East Sussex, UK.

Fukushima J, Fukushima K, Chiba T, Tanaka S, Yamashita I, Kato M (1988). Disturbances of voluntary control of saccadic eye movements in schizophrenic patients. Biological Psychiatry 23, 670-677.

Fukushima J, Fukushima K, Miyasaka K, Yamashita I (1994). Voluntary control of saccadic eye movement in patients with frontal cortical lesions and parkinsonian patients in comparison with that in schizophrenics. Biological Psychiatry 36, 21-30.

Fukushima J, Fukushima K, Morita N, Yamashita I (1990a). Further analysis of the control of voluntary saccadic eye movements in schizophrenic patients. Biological Psychiatry 28, 943-958.

Fukushima J, Morita N, Fukushima K, Chiba T, Tanaka S, Yamashita I (1990b). Voluntary control of saccadic eye movements in patients with schizophrenic and affective disorders. Journal of Psychiatric Research 24, 9-24.

Gottesman II, Gould TD (2003). The endophenotype concept in psychiatry: etymology and strategic intentions. American Journal of Psychiatry 160, 636-645.

Gould TD, Gottesman II (2005). Psychiatric endophenotypes and the development of valid animal models. Genes, Brain, and Behavior 5, 113-119.

Hutton SB, Cutherbert I, Crawford TJ, Kennard C, Barnes TRE, Joyce EM (2001). Saccadic hypometria in drug-naïve and drug-treated schizophrenic patients: a working memory deficit? Psychophysiology 38, 125-132.

Karoumi B, Saoud M, d'Amato T, Rosenfeld F, Denise P, Gutknecht C, Gaveau V, Beaulieu F-E, Dalery J, Rochet T (2001). Poor performance in smooth pursuit and antisaccadic eye-movement tasks in healthy siblings of patients with schizophrenia. Psychiatry Research 101, 209-219.

Karoumi B, Ventre-Dominey J, Vighetto A, Dalery J, d'Amato T (1998). Saccadic eye movements in schizophrenic patients. Psychiatry Research 77, 9-19.

Korn H (2000). Schizophrenic vulnerability: a deficiency of the correlation between foveal perception and oculomotor proprioception? Medical Hypotheses 55, 245-252.
Krebs M-O, Gut-Fayand A, Amado I, Daban C, Bourdel M-C, Poirier M-F, Berthoz A (2001). Impairment of predictive saccades in schizophrenia. Neuroreport 12, 465-469.

Krebs M-O, Gut-Fayand A, Bourdel M-C, Dischamp J, Olie J-P (2000). Validation and factorial structure of a standardized neurological examination assessing neurological soft signs in schizophrenia. Schizophrenia Research 45, 245-260.

Levy DL, O'Driscoll G, Matthysse S, Cook SR, Holzman PS, Mendell NR (2004). Antisaccade performance in biological relatives of schizophrenia patients: a meta-analysis. Schizophrenia Research 71, 113-125.

McDowell JE, Brenner CA, Myles-Worseley M, Coon H, Byerley W, Clementz BA (2001). Ocular motor delayed-response task performance among patients with schizophrenia and their biological relatives. Psychophysiology 38, 153-156.

McDowell JE, Clementz BA (1996). Ocular-motor delayedresponse task performance among schizophrenia patients. Neuropsychobiology 34, 67-71.

McDowell JE, Clementz BA (2001). Behavioral and brain imaging studies of saccadic performance in schizophrenia. Biological Psychology 57, 5-22.

Müller N, Riedel M, Eggert T, Straube A (1999). Internally and externally guided voluntary saccades in unmedicated and medicated schizophrenic patients. Part II. Saccadic latency, gain, and fixation suppression errors. European Archives of Psychiatry and Clinical Neuroscience 249, 7-14.

Nürnberger JI, Blehar MC, Kaufmann CA, York-Cooler C, Simpson SG, Harkavy-Friedman J, Severe JB, Malaspina D, Reich R (1994). Diagnostic Interview for Genetic Studies: rationale, unique features and training. Archives of General Psychiatry 51, 849-859.

Park S, Holzman PS, Goldman-Rakic PS (1995). Spatial working memory deficits in the relatives of schizophrenic patients. Archives of General Psychiatry 52, 821-828.

Pierrot-Deseilligny C (1994). Saccade and smooth-pursuit impairment after cerebral hemispheric lesions. European Journal of Neurology 34, 121-134.

Pierrot-Deseilligny C, Muri RM, Ploner CJ, Gaymard B, Demeret T, Rivaud-Pechoux S (2003). Decisional role of the dorsolateral prefrontal cortex in ocular motor behaviour. Brain 126, 1460-1473.

Ploner CJ, Gaymard BM, Rivaud-Péchoux S, PierrotDeseilligny C (2005). The prefrontal substrate of reflexive saccade inhibition in humans. Biological Psychiatry 57, 1159-1165.

Radant AD, Claypoole K, Wingerson DK, Cowley DS, Roy-Byrne PP (1997). Relationship between neuropsychological and oculomotor measures in schizophrenia patients and normal controls. Biological Psychiatry 42, 797-805.

Raemaekers M, Jansma JM, Cahn W, Van der Geest JN, van der Linden JA, Kahn RS, Ramsey NF (2002). Neuronal substrate of the saccadic inhibition deficit in schizophrenia investigated with 3-dimensional event-related functional magnetic resonance imaging. Archives of General Psychiatry 59, 313-320. 
Raemaekers M, Ramsey NF, Vink M, van den Heuvel MP, Kahn RS (2006). Brain activation during antisaccades in unaffected relatives of schizophrenic patients. Biological Psychiatry 59, 530-535.

Reilly J, Harris M, Keshavan M, Sweeney JA (2005). Abnormalities in visually guided saccades suggest corticofugal dysregulation in never-treated schizophrenia. Biological Psychiatry 57, 145-154.

Reulen J, Marcus J, Koops D, de Vries FR, Tiesinga G, Boshuizen K, Bos JE (1988). Precise recording of eye movement: the IRIS technique, Part 1. Medical and Biological Engineering and Computers 26, 20-26.

Reuter B, Herzog E, Kathmann N (2006). Antisaccade performance of schizophrenia patients: evidence of reduced task-set activation and impaired error detection. Journal of Psychiatric Research 40, 122-130.

Rijcken C, Monster T, Brouwers J, Lolkje T, de Jong-van den Berg W (2003). Chlorpromazine equivalents versus defined daily doses: how to compare antipsychotic drug doses? Journal of Clinical Psychopharmacology 23, 657-659.

Ross RG, Harris JG, Olincy A, Radant A, Adler LE, Freedman R (1998). Familial transmission of two independent saccadic abnormalities in schizophrenia. Schizophrenia Research 30, 59-70.

Schwartz BD, O'Brien BA, Evans WJ, McDermott DE, Sautter FJ, Winstead DK (1995). Abnormal saccadic eye movements associated with positive family history of schizophrenics. Biological Psychiatry 38, 487-491.

Sereno AB, Holzman PS (1995). Antisaccades and smooth pursuit eye movements in schizophrenia. Biological Psychiatry 37, 394-401.

Spitzer RL, Williams JBW, Gibbon M, First MB (1990). Structured Clinical Interview for DSM-III-R - Non-Patient Edition, Version 1.0. American Psychiatric Press: Washington, DC. 